\title{
The Projection of Individual Axons from the Parabrachial Region of the Brain Stem to the Dorsal Lateral Geniculate Nucleus in the Cat
}

\author{
Daniel J. Uhlrich, Josephine B. Cucchiaro, and S. Murray Sherman \\ Department of Neurobiology and Behavior, State University of New York at Stony Brook, Stony Brook, New York 11794
}

In mammals, the retinogeniculocortical pathway is the primary afferent route to visual cortex. The flow of information along this pathway can be modulated at the thalamic level (i.e., at the lateral geniculate nucleus) as a function of arousal, attention, and phenomena such as eye movements. Physiological studies indicate that an important source of this state-dependent influence on geniculate neuronal responsiveness is the parabrachial region of the brain stem. We used the anterograde tracer Phaseolus vulgaris leucoagglutinin to study the anatomical connections between the parabrachial region and the lateral geniculate nucleus. Labeled parabrachial axons are found throughout the thalamus, including all laminae of the lateral geniculate nucleus, the lateral posterior-pulvinar complex, the ventral lateral geniculate nucleus, the perigeniculate nucleus, and the reticular nucleus of the thalamus. Within these nuclei, the axons exhibit sporadically branched terminal arbors with boutons mostly en passant. Serial reconstructions indicate that individual parabrachial axons that innervate the lateral geniculate nucleus may terminate in other visual thalamic nuclei as well, but not in thalamic nuclei that subserve other modalities. Finally, the labeled parabrachial axons are morphologically heterogeneous; they differ in their innervation targets, terminal arbor shape, and the size spectrum of their boutons. These data suggest that this portion of the ascending parabrachial pathway, which may be functionally diverse, coordinates the responsiveness of the varied thalamic nuclei involved with vision.

In mammals, the retinogeniculocortical pathway is the primary route along which visual information travels to the cerebral cortex. Neural circuitry within the lateral geniculate nucleus along this route seems to gate the flow of visual information, and this modulation may reflect different behavioral states such as arousal, attention, and eye movements (for reviews, see Singer, 1977; Burke and Cole, 1978; Sherman and Koch, 1986). An important extrinsic source of this modulation is provided by cholinergic neurons located in the parabrachial rcgion of the brain stem, ${ }^{1}$ which is located within and dorsal to the brachium

Received Nov. 9, 1987; revised Mar. 24, 1988; accepted May 4, 1988

This work was supported by NIH Grants EY06610, EY03604, and EY03038. We thank Karen Manning for her helpful discussion on this manuscript and Joan Sommermeyer and Rachel Skovira for their excellent technical assistance.

Correspondence should be addressed to Dr. D. J. Uhlrich at the above address.

' There is some confusion concerning the terminolugy used to identify the predominantly cholinergic cell group in and around the brachium conjunctivum that projects to the visual thalamus. Recent studies in the rat have named this cell group the pedunculopontinc tegmental nucleus (Hallanger et al., 1987; Rye et al., 1987). In the cat, the best candidate for the homolog has been referred to as area $\mathrm{X}$ and the parabrachial nucleus (Sakai, 1980), the peribrachial nucleus (Smith et conjunctivum. Stimulation of this region typically increases excitability of geniculate relay cells (Singer, 1977; Burke and Cole, 1978; Sherman and Koch, 1986). Unfortunately, rather little is known about the anatomical basis of this brain-stem influence. In fact, it is not even clear whether ascending parabrachial axons innervate the lateral geniculate nucleus directly or whether they affect geniculate relay cells indirectly through intermediaries, such as the visual cortex or perigeniculate nucleus, which also innervate these relay cells. That is, evidence that parabrachial cells can be retrogradely labeled or antidromically activated from the lateral geniculate nucleus (Leger et al., 1975; Sakai and Jouvet, 1980; Ahlsen and Lo, 1982; Ahlsen, 1984; Hughes and Mullikin, 1984; De Lima and Singer, 1987) might reflect labeling or activation of fibers en route to other terminal destinations.

Recently, Stichel and Singer (1985) used choline acetyltransferase immunohistochemistry to identify cholinergic axons within the lateral geniculate and perigeniculate nuclei, but they were unable from this material to describe the terminal arbors of individual cholinergic axons. Also, the origin of their labeled fibers is somewhat ambiguous. For example, of the neurons in the brain stem that innervate the lateral geniculate nucleus, many in the parabrachial region are not cholinergic, while roughly half in the locus coeruleus are cholinergic (De Lima and Singer, 1987). For these reasons, in this and the following paper (Cucchiaro et al., 1988), we used the anterograde tracer Phaseolus vulgaris leucoagglutinin (PHA-L; see Gerfen and Sawchenko, 1984) in the cat to visualize individual axons projecting from the parabrachial region to the lateral geniculate nucleus and other thalamic nuclei. Preliminary results have been presented previously in abstract form (Uhlrich et al., 1985).

\section{Materials and Methods}

Adult cats were deeply anesthetized with intravenous injections of sodium pentobarbital (initially $15 \mathrm{mg} / \mathrm{kg}$, with $5-10 \mathrm{mg}$ supplements as needed), placed in a stereotaxic apparatus, and prepared for surgery with sterile precautions. We administered atropine sulfate $(0.15-0.20 \mathrm{mg})$ intramuscularly to reduce salivation. We continuously monitored heart rate and maintained the rectal temperature at $38^{\circ} \mathrm{C}$ via a feedbackcontrolled heating blanket. Wound edges and pressure points were periodically infused with $2 \%$ lidocaine, and the corneas were covered by contact lenses filled with ophthalmic solution. We then made a craniotomy and reflected the dura to allow penetration of the electrodes.

Anterograde tracing with PHA-L. Five cats were used. We delivered

al., 1988), and the parabrachial area of the central tegmental field (De Lima and Singer, 1987). However, these regions in the cat do not seem precisely homologous to the rat's pedunculopontine tegmental nucleus because all of the component cells in the rat are cholinergic (Rye et al., 1987), while a substantial minority of those in the cat appear to be noncholinergic (De Lima and Singer, 1987; Smith et al., 1988). For this reason and in order to be more consistent with prior terminology for the cat, we are calling this area the parabrachial region.

Copyright (c) 1988 Society for Neuroscience $0270-6474 / 88 / 124565-11 \$ 02.00 / 0$ 
the PHA-L to be injected in a glass micropipette $(1.0 \mathrm{~mm}$ capillary tubing). We first broke the micropipette's tip to an internal diameter of $10-15 \mu \mathrm{m}$ and then backfilled it with a $2.5 \%$ solution of PHA-L ( $5 \mathrm{mg} /$ $200 \mu \mathrm{l}$; Vector Labs) in $0.05 \mathrm{M}$ sodium phosphate buffer (pH 7.4). Once the micropipette was in place, PHA-L was injected iontophoretically with a 3-5 $\mu \mathrm{A}$ positive current at $0.07 \mathrm{~Hz}$ for $15-25 \mathrm{~min}$. Despite the relatively large diameter of the micropipette tips, the impedance of the electrodes so constructed was sufficiently high (up to $320 \mathrm{M} \Omega$ ) that the delivery of $5 \mu \mathrm{A}$ of current typically required $250-1600 \mathrm{~V}$.

In Horsely-Clark coordinates, parabrachial cells that project to the lateral geniculate nucleus are found rostrocaudally from $\mathrm{A} 2$ to $\mathrm{P} 2$ and mediolaterally from L1.5 to L4 (Berman, 1968; Leger et al., 1975; Ahlsen and Lo, 1982; see also Fig. $1 A$ ). While this is a fairly large target in the horizontal plane, the vertical extent of these cells, from coordinates D-2 to D-1, provides little margin for error. Consequently, while we sometimes used stereotaxic coordinates to place the tip of the PHA-L electrode in the brain, we often used electrophysiological criteria for more accurate localization. In these latter cases, the target area was first located with a low-impedance electrode, which consisted of another micropipette filled with $3 \mathrm{M} \mathrm{KCl}$. We then positioned the PHA-L electrode in the same coordinates for the injection. Because we could record field potentials and occasional single units through the PHA-L electrode, some of the physiological landmarks were reconfirmed. We have had success using the surface of the superior colliculus as a landmark and making our target $7.5-8.0 \mathrm{~mm}$ deep to that point. In 2 other cats, the brachium conjunctivum was stimulated with bipolar electrodes that were placed in the cerebellum. The potential generated by single shocks in the cerebellum was recorded through the $\mathrm{KCl}$ or PHA-L electrodes as they were lowered through the brain stem. The potential remained small until the electrode tip was approximately $8.0 \mathrm{~mm}$ below the superior colliculus, at which point a sharp increase in amplitude was observed. This indicated that the electrode entered the brachium conjunctivum. We placed the PHA-L injection $0.5 \mathrm{~mm}$ above this position. On occasion, the tentorium advanced far enough rostrally to prevent a direct vertical approach. For these, we extended the craniotomy anteriorly and approached the parabrachial region at a $20^{\circ}$ angle to the vertical.

After a $7-10 \mathrm{~d}$ survival period, the cat was reanesthetized, given heparin, and perfused transcardially with a brief saline rinse (1 $\mathrm{min})$ and then with 2 fixative solutions: 1 liter of $4 \%$ paraformaldehyde and $0.1 \mathrm{~m}$ acetate buffer ( $\mathrm{pH} 6.5$ ) followed by 1 liter of $4 \%$ paraformaldehyde and $0.05 \%$ glutaraldehyde in $0.05 \mathrm{~m}$ borate buffer ( $\mathrm{pH} 9.5$ ). The brain was removed from the skull, and blocks of tissue containing the injection site in the brain stem and the ipsilateral thalamus were postfixed overnight $\left(12-18 \mathrm{hr}\right.$ at $\left.4^{\circ} \mathrm{C}\right)$ in $20 \%$ sucrose dissolved in the second fixative. The next day, we cut $40-\mu \mathrm{m}$-thick coronal sections on a freezing microtome. The sections were preincubated with Triton $\mathrm{X}-100$ and reacted for PHA-L. For this reaction, we used the immunoperoxidase procedure based on the biotin-avidin system (ABC kit from Vector Labs) as described by Gerfen and Sawchenko (1984). The peroxidase was visualized with diaminobenzidine and cobalt chloride (Adams, 1977). All steps were carricd out at room temperaturc.

We then mounted the sections onto glass slides for examination under the light microscope. All reconstructions and measurements were done under oil-immersion optics at a magnification of 500 or 1000 using a camera lucida attachment. We did not correct our morphological analyses for tissue shrinkage.

Thus far, we have chosen to reconstruct serially all axons that were well labeled and could be easily followed from section to section. In most cases, we attempted to reconstruct all processes from a single axon to their termination. Termination of a process was determined when it ended with a bouton and could not be followed into a subsequent section. At each termination, we have also examined the corresponding areas in 2-3 tissue sections on each side of that termination. This precluded our incorrectly classifying a process as terminated when, in fact, it had abruptly shifted direction and crossed perpendicularly through adjacent sections, thereby rendering it difficult to visualize. Processes that we know are not fully reconstructed have been identified as such in the figures.

Retrograde tracing with HRP. HRP was injected into the lateral geniculate nucleus on one side in each of 2 cats. The injections were made via glass micropipette electrodes that had tip diameters of $7-11 \mu \mathrm{m}$ and were filled with $4 \% \mathrm{HRP}$ in $0.2 \mathrm{M}$ Tris-buffered $\mathrm{KCl}$. We lowered these electrodes into the brain until we recorded visually driven activity from geniculate neurons. We followed the progression of ocular dominance changes through the A-laminae until we encountered contralaterally driven units in lamina C. At this point, HRP was iontophoretically injected via a $5 \mu \mathrm{A}$ current at $0.1 \mathrm{~Hz}$ for $15 \mathrm{~min}$. We then retracted the electrode to perform similar injections separately in laminae A1 and A. Two such penetrations were made into the target lateral geniculate nucleus of each cat.

After a $24 \mathrm{hr}$ survival, the cat was decply reancsthetized, given heparin as noted above, and perfused transcardially with 2 liters of $1 \%$ paraformaldehyde and $2 \%$ glutaraldehyde in $0.1 \mathrm{M}$ phosphate buffer $(\mathrm{pH}$ 7.4) followed by $500 \mathrm{ml}$ of $10 \%$ sucrose in buffer. We then sectioned the brains frozen in the coronal plane at $100 \mu \mathrm{m}$. We reacted the sections with $o$-dianisidine (de Olmos, 1977) to visualize the injection sites and retrogradely labeled neurons in the brain stem.

\section{Results}

Figure 1 illustrates 2 series of sections running through the brain stem from caudal at the top to rostral at the bottom. Figure $1 \mathrm{~A}$ illustrates the cells in the parabrachial region that were retrogradely labeled after injections of HRP into the lateral geniculate nucleus (see also Leger et al., 1975; Ahlsen and Lo, 1982; Hughes and Mullikin, 1984). These cells were the target of our PHA-L injections. The most caudally located cells are in the locus coeruleus, and slightly rostral and lateral to these are cells in the parabrachial region. Caudally in the brain stem, these 2 cell groups are spatially separate, but they merge as they extend rostrally. An analogous conclusion can be drawn from recent immunohistochemical evidence in the cat (De Lima and Singer, 1987; Smith et al., 1988): Cholinergic and noradrenergic neurons that innervate the lateral geniculate nucleus overlap considerably, particularly in the rostral brain stem.

Figure $1 B$ demarcates 1 of 3 examples of a PHA-L injection site that occupies the entire rostrocaudal extent of the lateral portion of the parabrachial region. In 2 other cats, we made injections that were limited to the caudal parabrachial region (e.g., the third most caudal section in Fig. 1A). All of our injections spared the medial cell groups (i.e., the locus coeruleus and dorsal raphe) that innervate the lateral geniculate nucleus. With our injection parameters and survival times (see Materials and Methods), we find no evidence that PHA-L is taken up by fibers of passage, since we find no labeled processes in the lateral geniculate nucleus following control injections in the optic tract or radiations (see also Gerfen and Sawchenko, 1984). Furthermore, PHA-L injections into the brain stem that missed the target indicated in Figure $1 A$ do not result in the labeling of axons afferent to the lateral geniculate nucleus. However, with different injection parameters and shorter survival times, we occasionally see evidence that fibers of passage can be labeled for short distances $(<4 \mathrm{~mm})$, but these distances are insufficient to reach the thalamus from the parabrachial region. We thus conclude that the labeled axons in our material arise from the parabrachial cell group shown in Figure $1 \mathrm{~A}$.

These labeled axons and their terminal boutons are sparsely present in all visual regions of the thalamus, including all of the geniculate laminae, the lateral posterior-pulvinar complex, the ventral lateral geniculate nucleus, the perigeniculate nucleus, and the nearby thalamic reticular nucleus. (Unless stated otherwise, all references to the "lateral geniculate nucleus" refer to the dorsal lateral geniculate nucleus.) The morphology of labeled terminal arbors is similar in these different thalamic regions, although terminal labeling is most dense in the lateral posteriorpulvinar complex and ventral lateral geniculate nucleus. Furthermore, while we have not yet analyzed in any detail the labeled terminal arbors in nonvisual thalamic nuclei (e.g., the 
A

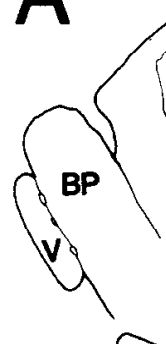<smiles>C1=C[Bi]C1</smiles>

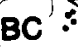<smiles>C1CCCCC1</smiles><smiles>C1CCCC1</smiles>

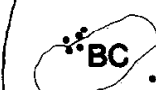<smiles>I[I-]I(I)I</smiles>

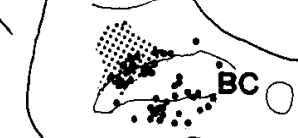
$\checkmark \sqrt{c}$

\section{$3 \mathrm{~mm}$}

Figure 1. Tracings of coronal sections through the midbrain at intervals of $0.5 \mathrm{~mm}$; caudal at top of sequence and rostral at bottom. A, Location of neurons (filled circles) retrogradely labeled from the lateral geniculate nucleus. The stippled area in the thirdmost caudal section shows the approximate location of a PHA-L injection that was placed in another cat. This PHA-L injection was restricted to the caudal portion of the parabrachial region. $B$, Another PHA-L injection site (stippled areas). This injection involved most of the parabrachial region containing neurons that project to the lateral geniculate nucleus (compare with $A$ ). Abbreviations: $B C$, brachium conjunctivum; $B P$, brachium pontis; $I C$, inferior colliculus; $L C$, locus coeruleus; $P$, pyramidal tract; $P A G$, periaqueductal gray; $V$, cranial nerve $\mathrm{V}$. 


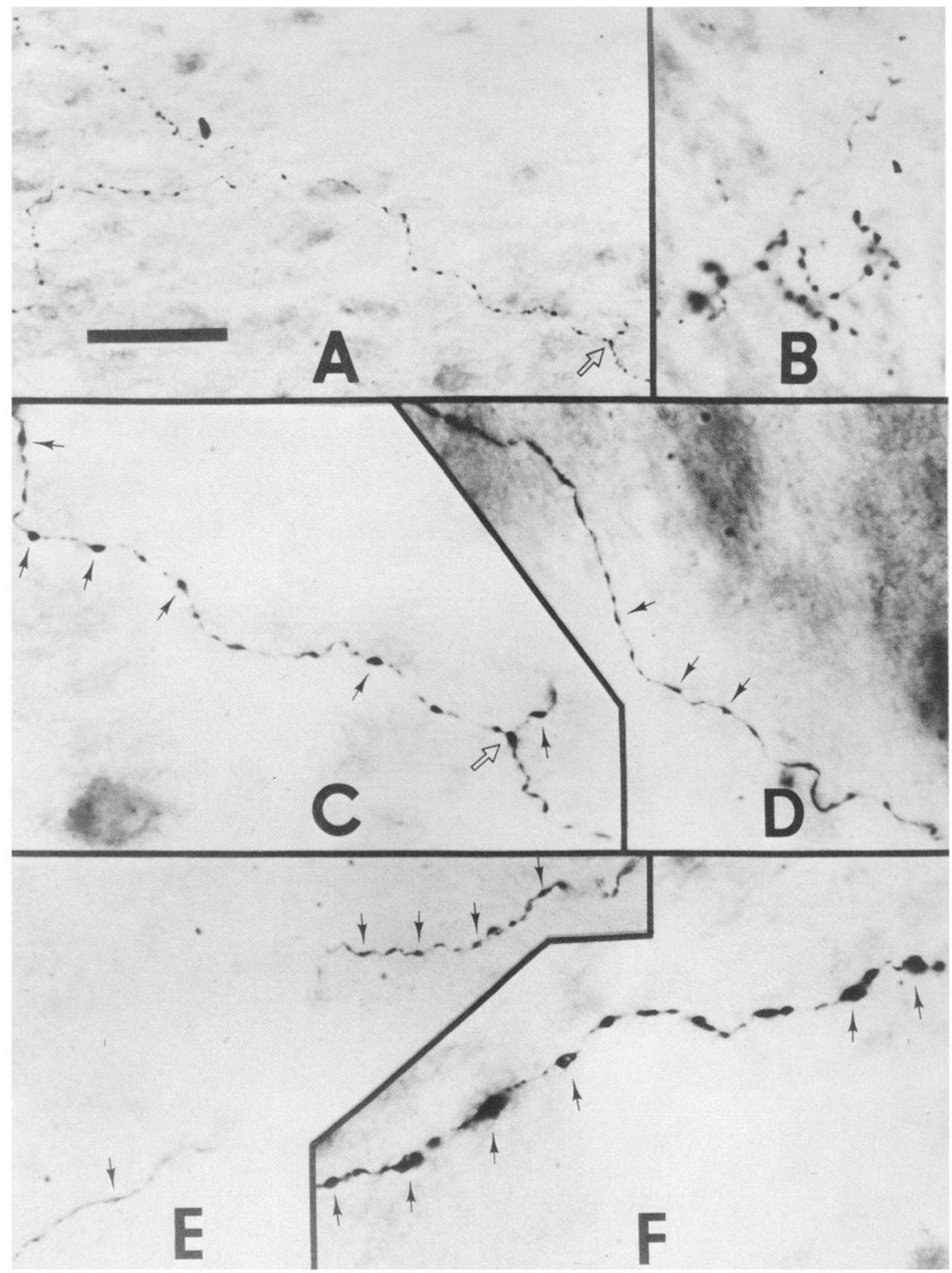

Figure 2. Photomicrographs of terminal arbors located in the lateral geniculate nucleus and labeled from the parabrachial region. The scale bar in $A$ represents $50 \mu \mathrm{m}$ for $A$ and $20 \mu \mathrm{m}$ for $B-F$. $A$, Labeled axon with numerous boutons en passant. The open arrow indicates a branch point that is shown again in a higher-power view indicated by the open arrow in $C$. B. Example of a cluster of boutons from a labeled parabrachial axon. $C$ and $F$, Axons with many larger boutons. $D$ and $E$, Axons with only smaller boutons. The filled arrows in $C-F$ point to representative boutons along the axons. 


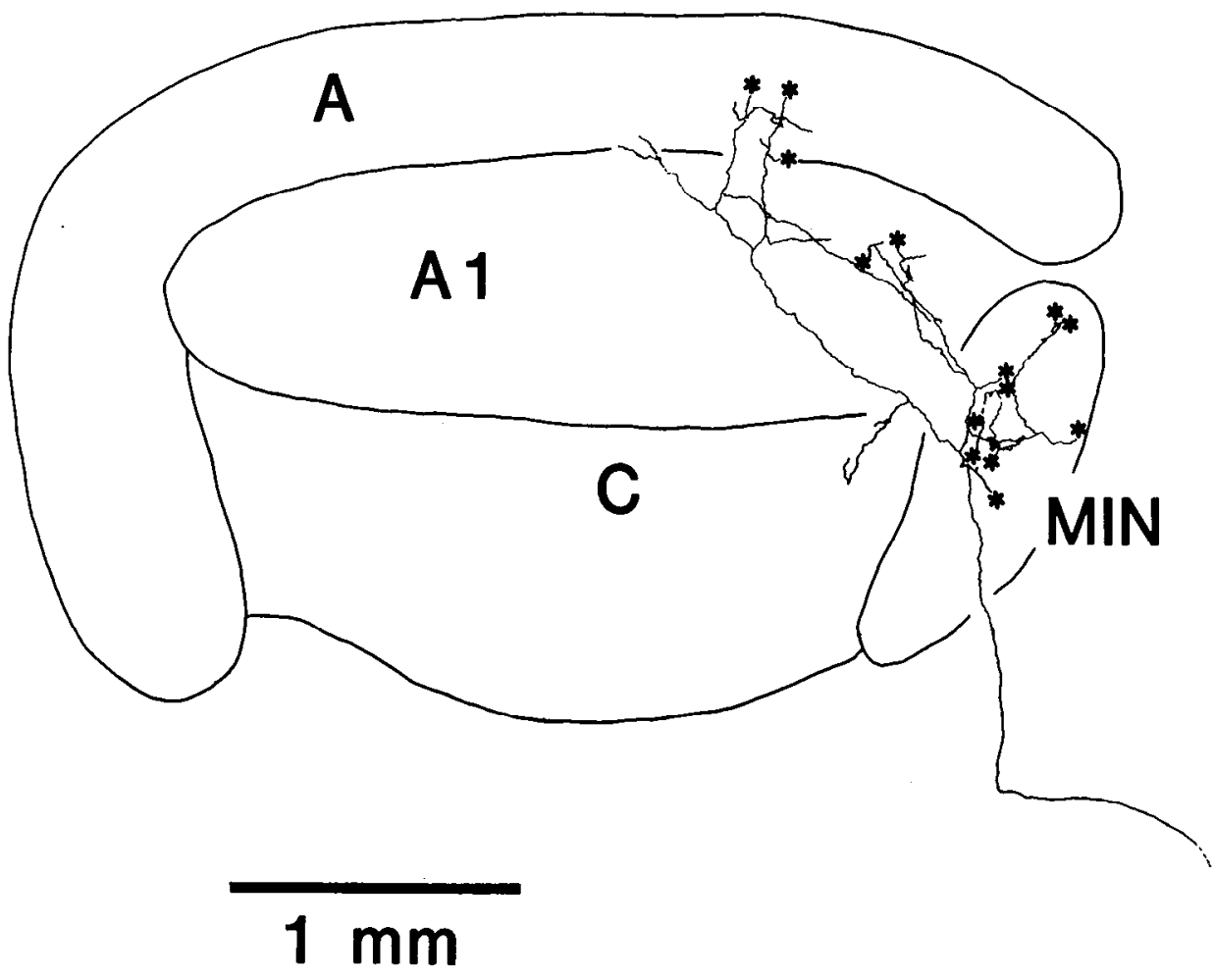

Figure 3. Arbor from a partially reconstructed parabrachial axon that has been reconstructed through more than $2 \mathrm{~mm}$ of tissue. All processes distal to the first bifurcation are laden with boutons. The diameter of the parent axon is $1.0 \mu \mathrm{m}$. In this and in Figures $4-7$, the reconstruction features the left lateral geniculate nucleus in the coronal plane. The asterisks denote cut endings of processes that have not been reconstructed to terminations. Abbreviations: $A$, lamina $\mathrm{A} ; A 1$, lamina $\mathrm{A} 1 ; C$, the C-laminae; $M I N$, the medial interlaminar nucleus. ventrobasal complex or medial geniculate nucleus), these arbors seem generally similar in morphological features to those innervating the lateral geniculate nucleus.

Figure 2 shows photomicrographs of axons and boutons in the lateral geniculate nucleus that were labeled from the parabrachial region. In the following paper (Cucchiaro et al., 1988), we demonstrate that such boutons are densely filled with syn- aptic vesicles and that many form conventional synapses onto geniculate relay neurons. Most of the labeled boutons are produced en passant, but they are occasionally appended to the axon by short stalks. A few axons branch and emanate small, local terminal arbors (e.g., Figs. 4, 5). Figure $2 B$ shows a cluster of labeled boutons, which is rare in the lateral geniculate nucleus but is more commonly found around the somata and proximal

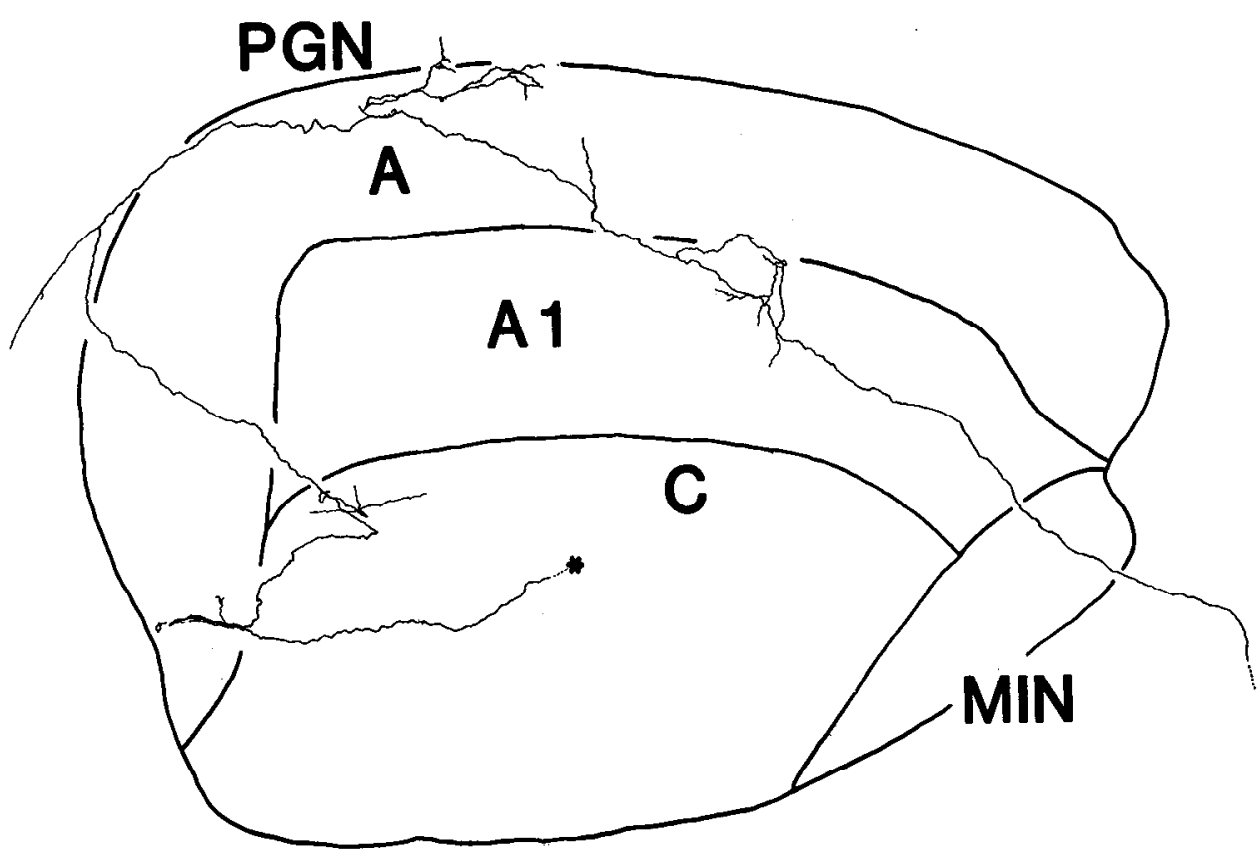

$1 \mathrm{~mm}$
Figure 4. Partially reconstructed axon with retinotopically diffuse innervation of the lateral geniculate nucleus. From its entry point at the medial interlaminar nucleus, the axon was followed rostrally for $2 \mathrm{~mm}$ to the terminal arbor in the dorsal region of lamina $\mathrm{A}$ and the perigeniculate nucleus $(P G N)$, then caudally for $1.5 \mathrm{~mm}$ to the arbor in lamina $\mathrm{C}$, and then rostrally again for $1 \mathrm{~mm}$ to the arbor in the lateral region of lamina A. All boutons in this terminal arbor are found on the small side branches. The parent axon was $0.5 \mu \mathrm{m}$ in diameter. 


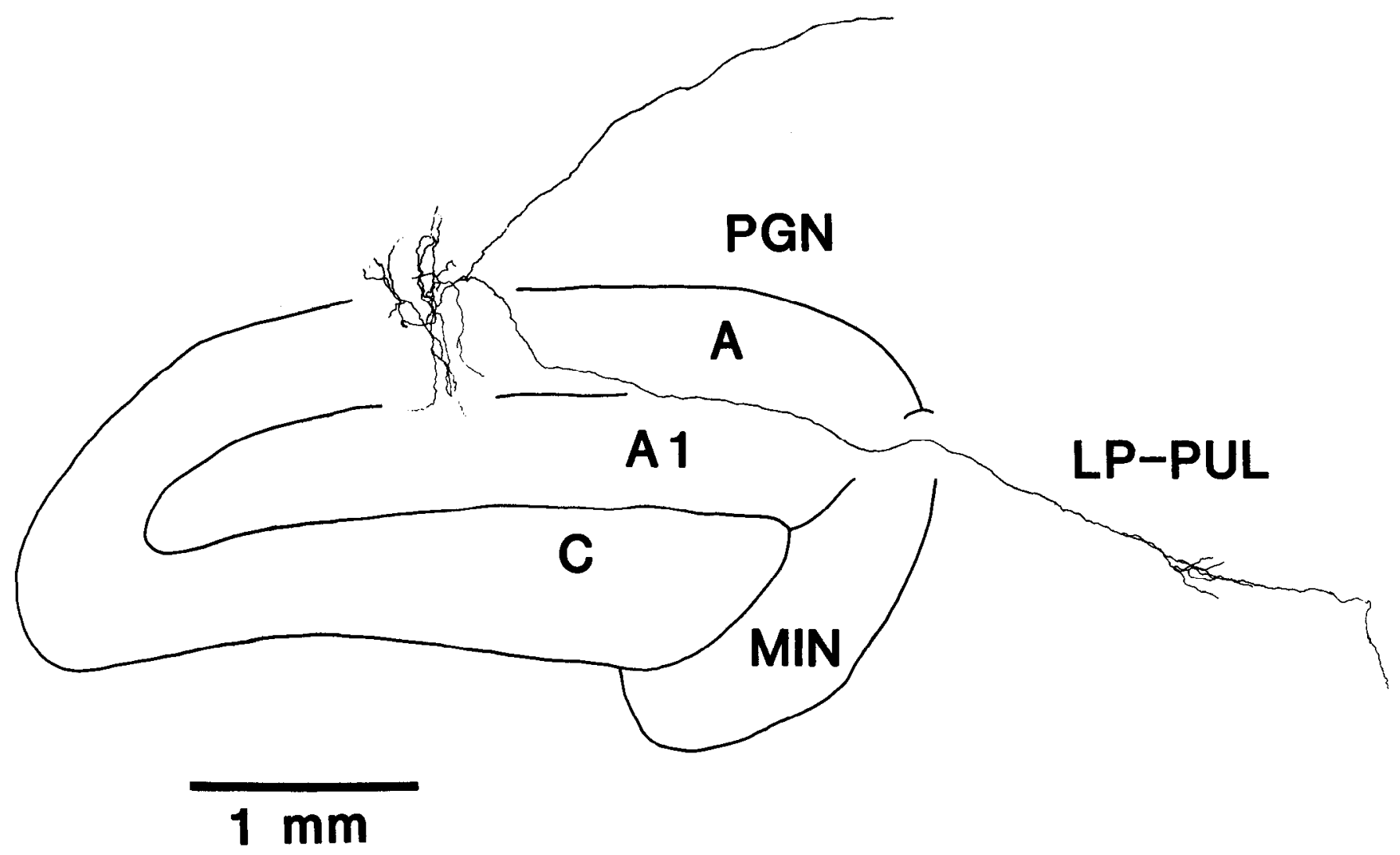

Figure 5. Axon with retinotopically restricted projection located within the lateral geniculate nucleus, perigeniculate nucleus, and lateral posteriorpulvinar complex $(L P-P U L)$. Within the lateral geniculate nucleus, the axon innervates lamina $\mathrm{A}$ and the interlaminar zone between laminae $\mathrm{A}$ and $\mathrm{Al}$ but does not enter lamina A1. We traced the parent axon, which was $0.5 \mu \mathrm{m}$ in diameter, for 3 mm caudomedially from the arbor in the lateral posterior-pulvinar complex and for $5 \mathrm{~mm}$ rostromedially from the arbor in lamina $\mathrm{A}$. In this entire span, we found no further axonal branching.

dendrites of perigeniculate cells (see also Stichel and Singer, 1985).

\section{Reconstruction of individual axons}

Because the labeled parabrachial axons are sparsely distributed and are densely labeled, we have been able to reconstruct a number of individual examples. We partially reconstructed over 40 labeled axonal processes coursing through the lateral geniculate nucleus and adjacent thalamic structures. Sixteen of these axons were reconstructed for a considerable extent, usually for more than $3 \mathrm{~mm}$. While most of these axons approach the lateral geniculate nucleus from a ventromedial or ventrolateral direction, often after a detour into the optic tract to run with retinogeniculate axons (see also Robertson, 1981), we have examples of parabrachial axons entering this nucleus from virtually all directions. Terminal arborization patterns are also varied, as illustrated by the examples in Figures 3-7.

Figure 3 represents the most common type of terminal arbor, for which the parent axon enters the lateral geniculate nucleus from a ventromedial direction and arborizes over a large portion of this nucleus in a meshlike fashion. This axon displays occasional bouton clusters in the medial interlaminar nucleus, and all axon branches distal to the first bifurcation are laden with boutons. Although the terminal arbor is not fully reconstructed, it runs rostrolaterally across more than $2 \mathrm{~mm}$ of the lateral geniculatc nuclcus, which is a much larger retinotopic representation (Sanderson, 1971) than exists for individual retinal, cor- tical, or perigeniculate afferents to the lateral geniculate nucleus (Updyke, 1975; Bowling and Michael, 1984; Robson, 1984; Cucchiaro et al., 1985; Sur et al., 1987; Uhlrich et al., 1987).

Figure 4 illustrates another parabrachial axon with a diffuse innervation of the lateral geniculate nucleus. The axon enters the nucleus from a ventromedial direction. However, rather than being bouton rich throughout its termination, this example emits 6 widely spaced bouton clusters as it courses back and forth mediolaterally and rostrocaudally through more than 2 $\mathrm{mm}$ of the lateral geniculate nucleus. The terminal clusters are not retinotopically related, since they appear to innervate geniculate regions with visual field representations that differ by as much as $50^{\circ}$ (cf. Sanderson, 1971). The tortuous course of this axon exemplifies a fairly common property of brain-stem axons as gleaned from our material. This poses a formidable fiber of passage problem in the interpretation of studies of brainstem neurons based on retrograde transport of label or antidromic activation (e.g., Leger et al., 1975; Sakai and Jouvet, 1980; Ahlsen and Lo, 1982; Ahlsen, 1984; Hughes and Mullikin, 1984). That is, one can retrogradely label or antidromically activate a brain-stem neuron by injections of tracer or current along the cell's axon at a position that may not correspond closely to the axon's terminal arbor.

Figures 5 and 6 show labeled terminal arbors that are much more restricted than any suggested by prior studies of this pathway (e.g., Scheibel and Scheibel, 1958; Ahlsen, 1984). The axon in Figure 5 innervates retinotopically restricted portions of ge- 


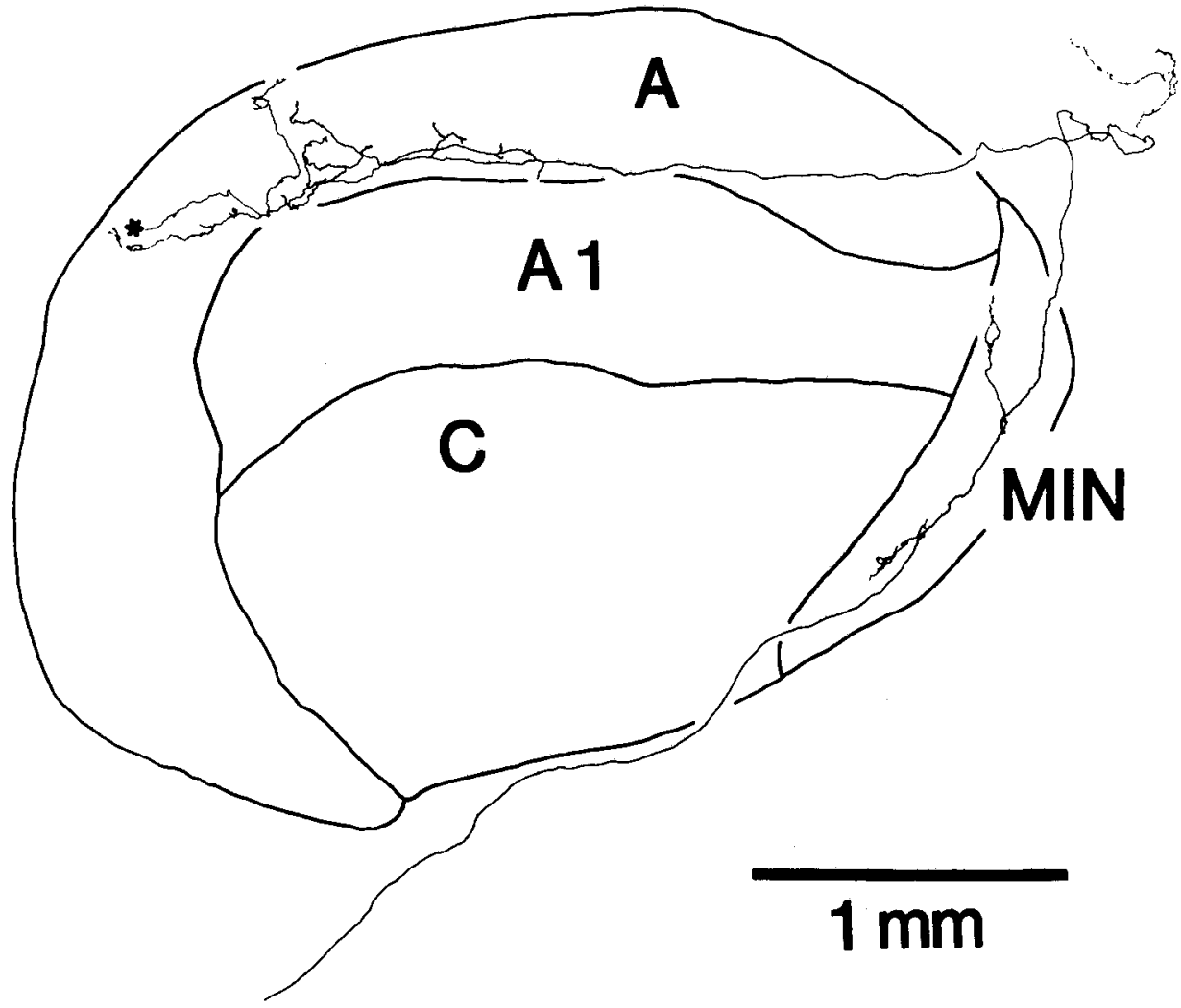

Figure 6. Axon that innervates lamina $A$, the medial interlaminar nucleus, and a region rostromedial to lamina $A$ that is probably the retinorecipient geniculate wing. The diameter of the parent axon was $0.5 \mu \mathrm{m}$. niculate lamina $\mathrm{A}$ and the perigeniculate nucleus, and it avoids lamina $\mathrm{A} 1$ and the C-laminae. Similarly, the terminations in the main geniculate laminae of the axon in Figure 6 are limited to lamina $\mathrm{A}$ and the medial interlaminar nucleus.

Figures 5-7 illustrate further that many parabrachial axons projecting to the lateral geniculate nucleus also terminate outside the cytoarchitectural boundaries of the nucleus. For example, the axon in Figure 5 also emits a small arbor in the lateral posterior-pulvinar complex, and even though it could not be traced to all its destinations, the distal end of this axon most certainly leads to another termination. The axons in Figures 6 and 7 innervate a region just medial to lamina $A$ that is either a visual portion of the pulvinar or the retinorecipient geniculate wing (Guillery et al., 1980; Leventhal, 1980). We also have observed axons that innervate both the ventral and dorsal lateral geniculate nuclei (not illustrated). Because the retinotopic maps of all these visual nuclei cannot be determined from anatomical material, it is unclear how well the retinotopic representations of terminal arbors are matched in these various thalamic nuclei.

Many, but not all, axons in our sample that innervate the lateral geniculate nucleus could be followed to other thalamic terminal zones. Conversely, some of the labcled axons that innervate the lateral posterior-pulvinar complex or ventral lateral geniculate nucleus fail to innervate the dorsal lateral geniculate nucleus. Thus far, all of our reconstructed axons that depart from the lateral geniculate nucleus terminate in other visual thalamic nuclei. We have traced some axons for more than 12 $\mathrm{mm}$ from their geniculate terminal zones, and none has branched to innervate a nonvisual thalamic structure, such as the ventrobasal complex or medial geniculate nucleus.

\section{Bouton size}

Not only do parabrachial axons vary with respect to the gross morphology of their terminal arbors, at least within regions of the thalamus that represent vision, but they also vary considerably in the sizes of the boutons they contain (Fig. 2). Figure 8 , which illustrates a representative series of histograms of bouton size within the lateral geniculate nucleus, shows that populations of boutons labeled from different injection sites and even boutons from different individual axons labeled from the same injection differ dramatically in size. For instance, Figure $8, D-I$ represents data from individually labeled axons, and bouton size distributions vary significantly among this group ( $p$ $<0.001, \chi^{2}$ test). Significant differences are also seen on many pairwise comparisons of these axons: The axons of Figure 8, $G$, $H$, are labeled from the same injection, yet the boutons of Figure $8 G$ are smaller on average than are those of Figure $8 H(p<$ 0.001 , Mann-Whitney $U$ test); likewise, the boutons of Figure $8 D$ are smaller than those of either Figure $8 E$ or Figure $8 F(p$ $<0.001$, Mann-Whitney $U$ test for each comparison), although all 3 axons are labeled from the same injection.

Finally, our data suggest that the larger boutons tend to be labeled from more rostral parabrachial regions. Figure $8 \mathrm{C}$ represents boutons pooled from several axons labeled from an injection limited to the caudal parabrachial region; all of the other histograms represent data from larger injections involving both caudal and rostral parabrachial regions. The boutons of Figure $8 C$ are smaller on average than the bouton populations represented by either Figure $8 B$, which represents another bouton population pooled from several axons, or Figure $8 A$, which in- 


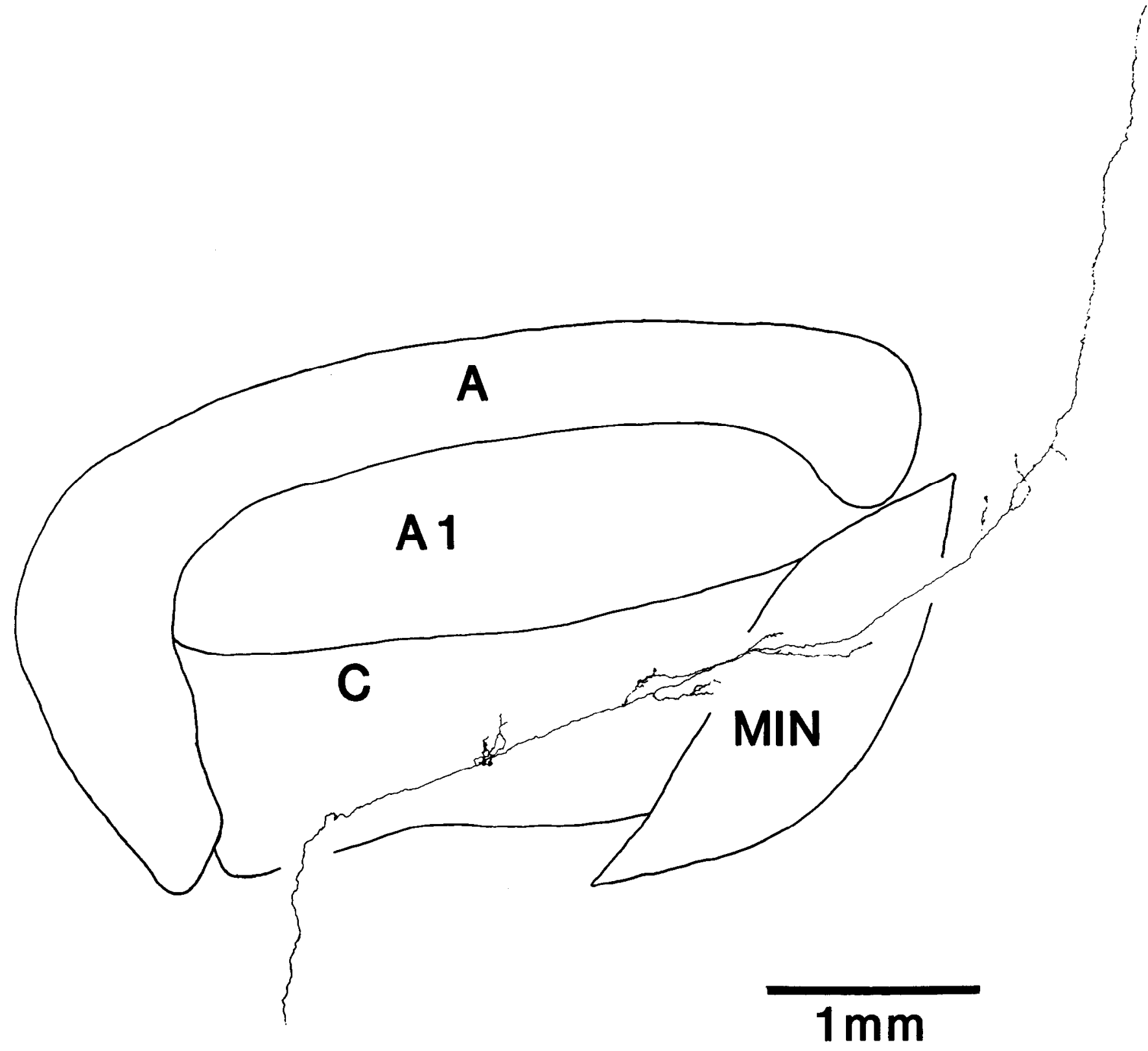

Figure 7. Parabrachial axon that travels in the optic tract and innervates the C-laminae, the medial interlaminar nucleus, and a region that is probably the geniculate wing. All processes distal to the second bifurcation in the C-laminae are laden with boutons. The diameter of the parent axon was $0.75 \mu \mathrm{m}$.

dicates the entire population of boutons shown in Figure 8, B$I(p<0.01$, Mann-Whitney $U$-test for both comparisons). One other injection limited to the caudal parabrachial region (not illustrated) also failed to label axons with larger boutons.

\section{Discussion}

We have used PHA-L as an anterograde tracer to visualize the ascending parabrachial projection to the dorsal lateral geniculate nucleus and other thalamic components of the central visual pathways. This is the first anterograde demonstration of the nature of this brain-stem projection at the single-axon level. We draw 3 main conclusions from this work. First, axons terminate throughout the thalamus, including all visual thalamic nuclei. Second, parabrachial axons that innervate the dorsal lateral geniculate nucleus usually innervate other visual thalamic nuclei as well, but they do not innervate thalamic nuclei that subserve other sensory modalities. Third, the ascending parabrachial projection is heterogeneous: Labeled axons vary in terms of their arbor morphology, including the size spectrum of their boutons, and their efferent targets.

\section{Possible role in modulation of geniculate relay cells}

There are several routes through which axons from the parabrachial region may affect the responsiveness of geniculate relay cells. All geniculate laminae receive input from parabrachial axons, and we demonstrate in the following paper (Cucchiaro ct al., 1988) that, at least in the A-laminae, these axons contact both relay cells and interneurons there. Parabrachial axons, thus, can directly modulate geniculate relay cells. Furthermore, through modulation of the interneurons, which are GABAergic neurons 


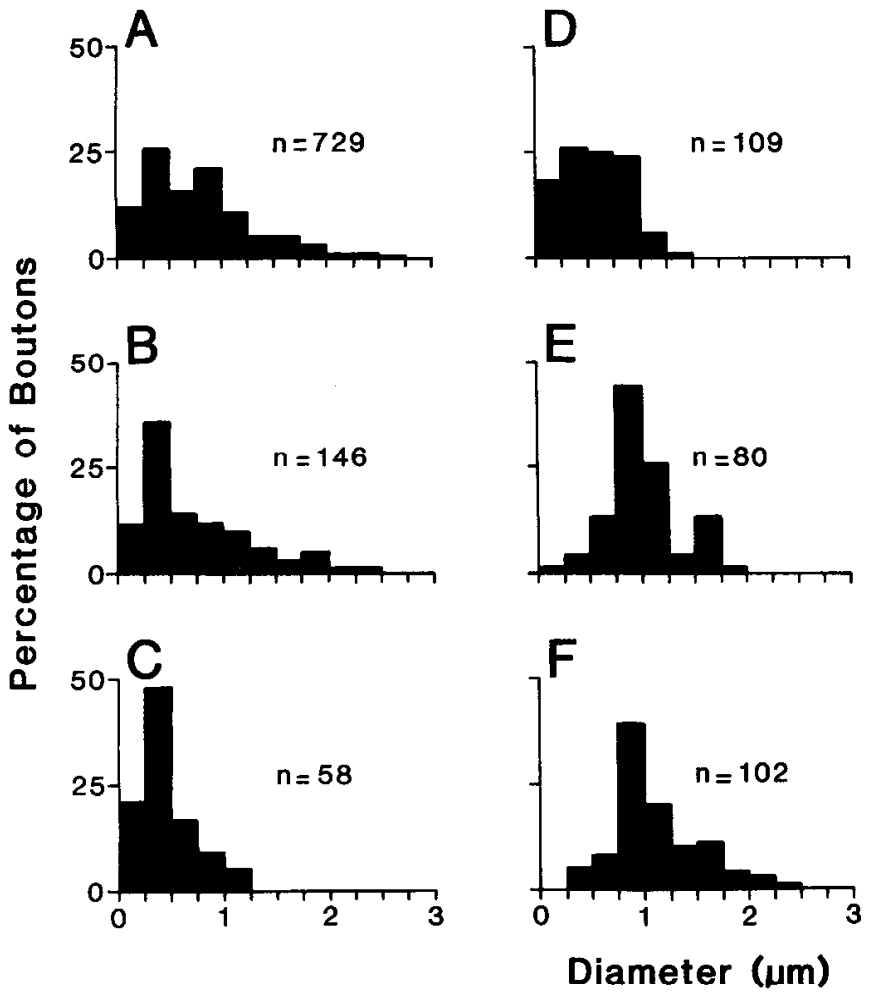

that innervate and influence relay cells (Fitzpatrick et al., 1984; Hamos et al., 1985; Montero, 1986), these parabrachial axons can indirectly modify relay cell responsiveness (Ahlsen et al., 1984). Another indirect route to modify relay cell responsiveness is suggested by parabrachial innervation of the perigeniculate nucleus. Perigeniculate neurons, which are also GABAergic (Fitzpatrick et al., 1984; Montero and Singer, 1984), project to the lateral geniculate nucleus to innervate neurons there (Cucchiaro et al., 1985; Uhlrich et al., 1987).

There obviously exist many neuronal circuits by which cells from the parabrachial region can modify retinogeniculocortical transmission (see Sherman and Koch, 1986). To add to this complexity, geniculate neurons may be able to respond in a variety of quite different manners to the same type of parabrachial input. For instance, $\mathrm{ACh}$, which seems to be the most common neurotransmitter used by the parabrachial axons that innervate the lateral geniculate nucleus (De Lima and Singer, 1987), can operate on geniculate neurons via at least 3 receptors, 2 muscarinic and 1 nicotinic (McCormick and Prince, 1987). The postsynaptic response would depend profoundly on the receptor present. We are thus far from any reasonable understanding of precisely how inputs from the parabrachial region function to control retinogeniculocortical transmission.

\section{Specificity of parabrachial innervation for the visual system}

Our reconstructions of individual parabrachial axons confirms the suggestion from electrophysiological studies based on antidromic activation that these axons can innervate more than one visual thalamic nucleus and that these axons are specific to the visual system. Sakai and Jouvet (1980) and Ahlsen (1984) reported that individual brain-stem axons could be antidromically activated from both the lateral geniculate nucleus and the visual representation within the pulvinar; furthermore, cells activated from the lateral geniculate nucleus could not be driven
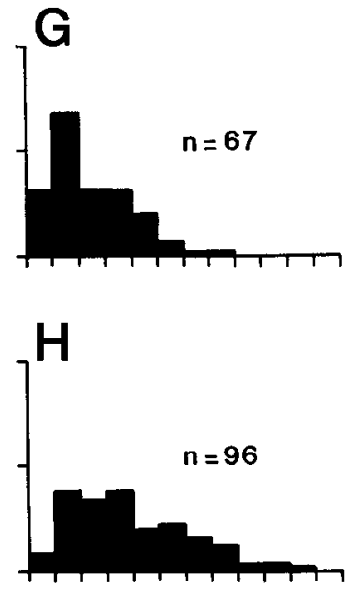

Figure 8. Distributions of bouton dianeters from labeled parabrachial axons; all of these boutons were located within the lateral geniculate nucleus. $A$, Pooled distribution for all boutons represented in $B-I . B$ and $C$, Distributions of randomly selected boutons labeled from injections illustrated in Figures 1, $B$ and $A$, respectively. $D-I$, Distributions for individual axons. The histograms in $D-F$ represent 3 axons labeled from the same injection. Those in $G$ and $H$ represent 2 other axons labeled from another single injection in a different cat (the same injection as in $B$, but $B, G$, and $I I$ represent different samples of boutons). Those in $I$ represent yet another axon from another cat. Histograms $E, D, I, G$, and $F$, represent the same axons as illustrated in Figures 3, $4,5,6$, and 7 , respectively. Numerous statistical differences are seen in many comparisons among these histograms (see text for examples).

from the somatosensory thalamus. Likewise, we observe axons that innervate the visual representation within the pulvinar (i.e., the lateral posterior-pulvinar complex), the perigeniculatc nucleus, and/or the ventral lateral geniculate nucleus in addition to the dorsal lateral geniculate nucleus. We never observed any parabrachial axon innervating one of these visual thalamic regions in addition to a nonvisual one, such as the medial geniculate nucleus or the ventrobasal complex.

Thus, parabrachial axons may help to coordinate the visual activity among different thalamic nuclei. We found no evidence to support the notion that the parabrachial region is involved in the integration of activity among various sensory modalities, at least at the thalamic level. Perhaps other regions of the brainstem reticular formation, such as the locus coeruleus or dorsal raphe nucleus, are involved in such transmodality integration. We hope to test that possibility with experiments similar to those described here by labeling these other nuclei with PHAL. Another prospect that requires additional testing is the possibility that, at least at the level of thalamus, other parabrachial axons are as specific for the somatosensory or auditory modalities as are those reconstructed in the present study for the visual modality.

\section{Heterogeneity among parabrachial axons}

Our final major conclusion is that axons labeled from the parabrachial region differ considerably in their projection to the lateral geniculate nucleus. We observed differences in the patterns of innervation to thalamic nuclei and in the detailed structure of the terminal arbors. Some axons are laden with boutons along their full extent in the lateral geniculate nucleus, while others produce boutons only on small side branches that emanate from the parent axon. Axons may also differ in the size spectrum of their boutons. Furthermore, it is not clear that the PHA-L method we have employed labels all cells at the injection 
site or all classes of brain-stem axon in the lateral geniculate nucleus, so there may be even more heterogeneity present in this pathway than we have described. Despite this apparent heterogeneity, our sample is presently too small to rule out the possibility that we have observed morphological variation along a continuum within a single axonal class.

It nonetheless seems worthwhile to consider the prospect that some of the variability may indeed reflect distinctly different classes among the axons' cells of origin. For example, one group of parabrachial cells may affect general arousal or attention (see Singer, 1977; Sherman and Koch, 1986) and might therefore be expected to innervate the lateral geniculate nucleus diffusely (e.g., our examples in Figs. 3 and 4; see also Ahlsen, 1984). Another subset of parabrachial neurons, those with localized terminal arbors in the lateral geniculate nucleus (e.g., our examples in Figs. 5 and 6), might subserve a more specific or retinotopically restricted function. Such a function might be the coordination of visual activity with eye movements or some other oculomotor event that has a retinotopic consequence. In support of this idea, Singer and Bedworth (1974) demonstrated that electrical stimulation of sites in the brain stem and the occurrence of eye movements have similar facilitatory effects on geniculate relay cells. Finally, the morphological variability in our axon sample could be related to the neurotransmitters used by the different axons. Immunocytochemical studies indicate that most neurons of the parabrachial region contain either ACh or noradrenaline and that both cholinergic and noradrenergic fibers traverse the lateral geniculate nucleus (Maeda et al., 1973; Kromer and Moore, 1980; Kimura et al., 1981; De Lima and Singer, 1987). It remains for future experiments to determine how the variations in morphology relate to different neurotransmitters or other functional attributes of ascending parabrachial axons.

\section{References}

Adams, J.C. (1977) Technical considerations on the use of horseradish peroxidase as a neuronal marker. Neuroscience 2: 141-145.

Ahlsen, G. (1984) Brainstem neurons with differential projections to functional subregions of the dorsal lateral geniculate complex in the cat. Neuroscience 12:817-838.

Ahlsen, G., and F.-S. Lo (1982) Projection of brainstem neurones to the perigeniculate nucleus and the lateral geniculate nucleus in the cat. Brain Res. 238: 433-438.

Ahlsen, G., S. Lindstrom, and F.-S. Lo (1984) Inhibition from the brainstem of inhibitory interneurones of the cat's dorsal lateral geniculate nucleus. J. Physiol. (Lond.) 347: 593-609.

Berman, A. I. (1968) The Brain Stem of the Cat. A Cytoarchitectonic Atlas with Stereotaxic Coordinates, University of Wisconsin Press, Madison, WI.

Bowling, D. B., and C. R. Michael (1984) Terminal patterns of single, physiologically characterized optic tract fibers in the cat's lateral geniculate nucleus. J. Neurosci. 4: 198-216.

Burke, W., and A. M. Cole (1978) Extraretinal influences on the lateral geniculate nucleus. Rev. Physiol. Biochem. Pharmacol. 80: 105-166.

Cucchiaro, J. B., D. J. Uhlrich, J. E. Hamos, and S. M. Sherman (1985) Perigeniculate input to the cat's lateral geniculate nucleus: A light and electron microscopic study of single, HRP filled cells. Soc. Neurosci. Abstr. 11:231.

Cucchiaro, J. B., D. J. Uhlrich, and S. M. Sherman (1988) Parabrachial innervation of the cat's lateral geniculate nucleus: An electron microscopic study using the tracer Phaseolus vulgaris leucoagglutinin (PHA-L). J. Neurosci. 8: 4576-4588.

De Lima, A. D., and W. Singer (1987) The brainstem projection to the lateral geniculate nucleus in the cat: Identification of cholinergic and monoaminergic elements. J. Comp. Neurol. 259: 92-121.

de Olmos, J. S. (1977) An improved HRP method for the study of central nervous connections. Exp. Brain Res. 29: 541-551.
Fitzpatrick, D., G. R. Penny, and D. E. Schmechel (1984) Glutamic acid decarboxylase-immunoreactive neurons and terminals in the lateral geniculate nucleus of the cat. J. Neurosci. 4: 1809-1829.

Gerfen, C., and P. E. Sawchenko (1984) An anterograde neuroanatomical tracing method that shows the detailed morphology of neurons, their axons and terminals: Immunohistochemical localization of an axonally transported plant lectin, Phaseolus vulyaris leucoagglutinin (pha-1). Brain Res. 290: 219-238.

Guillery, R. W., E. E. Geisert, Jr., E. H. Polley, and C. A. Mason (1980) An analysis of the retinal afferents to the cat's medial interlaminar nucleus and to its rostral thalamic extension, the "geniculate wing." J. Comp. Neurol. 194: 117-142.

Hallanger, A. E., A. I. Levey, H. J. Lee, D. B. Rye, and B. H. Wainer (1987) The origins of cholinergic and other subcortical afferents to the thalamus in the rat. J. Comp. Neurol. 262: 105-124.

Hamos, J. E., S. C. Van Horn, D. Raczkowski, D. J. Uhlrich, and S. M. Sherman (1985) Synaptic connectivity of a local circuit neurone in the lateral geniculate nucleus of the cat. Nature 317: 618-621.

Hughes, H. C., and W. H. Mullikin (1984) Brainstem afferents to the lateral geniculate nucleus of the cat. Exp. Brain Res. 54: 253-258.

Kimura, H., P. L. McGeer, J. H. Peng, and E. G. McGeer (1981) The central cholinergic system studied by choline acetyltransferase immunohistochemistry in the cat. J. Comp. Neurol. 200: 151-201.

Kromer, L. F., and R. Y. Moore (1980) A study of the organization of the locus coeruleus projections to the lateral geniculate nuclei in the albino rat. Neuroscience 5: 255-271.

Leger, L., K. Sakai, D. Salvert, M. Touret, and M. Jouvet (1975) Delineation of the dorsal lateral geniculate afferents from the cat brain stem visualised by the horseradish peroxidase technique. Brain Res. 93: $490-496$.

Leventhal, A. (1980) The afferent ganglion cells and cortical projections of the retinal recipient zone (RRZ) of the cat's "pulvinar complex." J. Comp. Neurol. 194: 535-554.

Maeda, T., C. Pin, D. Salvert, M. Ligier, and M. Jouvet (1973) Les neurones contenant des catecholamines du tegmentum pontique et leurs vois de projection chez le chat. Brain Res. 57: 119-152.

McCormick, D. A., and D. A. Prince (1987) Actions of acetylcholine in the guinea pig and cat medial and lateral geniculate nuclei, in vitro. J. Physiol. (Lond.) 392: 147-165.

Montero, V. M. (1986) Localization of gamma-aminobutyric acid (GABA) in type 3 cells and demonstration of their source to F2 terminals in the cat lateral geniculate nucleus: A Golgi-electron-microscopic GABA-immunocytochemical study. J. Comp. Neurol. 254: 228-245.

Montero, V. M., and W. Singer (1984) Ultrastructure and synaptic relations of neural elements containing glutamic acid decarboxylase (GAD) in the perigeniculate nucleus of the cat: A light and electron microscopic immunocytochemical study. Exp. Brain Res. 56: 115125.

Robertson, R. T. (1981) Projections from the pontine reticular formation to the lateral geniculate body: Autoradiographic demonstration of a pathway that could mediate pontine-geniculate-occipital waves. Exp. Neurol. 73: 576-581.

Robson, J. A. (1984) Reconstructions of corticogeniculate axons in the cat. J. Comp. Neurol. 225: 193-200.

Rye, D. B., C. B. Saper, H. J. Lee, and B. H. Wainer (1987) Pedunculopontine tegmental nucleus of the rat: Cytoarchitecture, cytochemistry, and some extrapyramidal connections of the mesopontine tegmentum. J. Comp. Neurol. 259: 483-528.

Sakai, K. (1980) Some anatomical and physiological properties of ponto-mesencephalic tegmental neurons with special reference to the PGO waves and postural atonia during paradoxical sleep in the cat. In The Reticular Formation Revisited, J. A. Hobson and M. A. B. Brazier, eds., pp. 427-447, Raven, New York.

Sakai, K., and M. Jouvet (1980) Brain stem PGO-on cells projecting directly to the cat dorsal lateral geniculate nucleus. Brain Res. 194: 500-505.

Sanderson, K. J. (1971) The projection of the visual field to the lateral geniculate and medial interlaminar nuclei in the cat. J. Comp. Neurol. 143: 101-118.

Scheibel, M. E., and A. B. Scheibel (1958) Structural substrates for integrative patterns in the brain stem reticular core. In Reticular Formation of the Brain, H. H. Jasper, ed., pp. 31-55, Little, Brown, Boston.

Sherman, S. M., and C. Koch (1986) The control of retinogeniculate 
transmission in the mammalian lateral geniculate nucleus. Exp. Brain Res. 63: 1-20.

Singer, W. (1977) Control of thalamic transmission by corticofugal and ascending reticular pathways in the visual system. Physiol. Rev. 57: 386-420.

Singer, W., and N. Bedworth (1974) Correlation between the effects of brain stem stimulation and saccadic eye movements on transmission in the cat lateral geniculate nucleus. Brain Res. 72: 185-202.

Smith, Y., D. Pare, M. Deschenes, A. Parent, and M. Steriade (1988) Cholinergic and non-cholinergic projections from the upper brainstem core to the visual thalamus in the cat. Exp. Brain Res. 70: 166-180.

Stichel, C. C., and W. Singer (1985) Organization and morphological characteristics of choline acetyltransferase-containing fibers in the visual thalamus and striate cortex of the cat. Neurosci. Lett. 53: 155160.
Sur, M., M. Esguerra, P. E. Garraghty, M. F. Kritzer, and S. M. Sherman (1987) Morphology of physiologically identified retinogeniculate Xand $\mathrm{Y}$-axons in the cat. J. Neurophysiol. 58: 1-32.

Uhlrich, D. J., J. B. Cucchiaro, and S. M. Sherman (1985) Terminal patterns of the brainstem projection to the lateral geniculate nucleus in the cat: An anterograde tracer study using Phaseolus vulgaris leucoagglutinin (PHA-L). Soc. Neurosci. Abstr. 11: 232.

Uhlrich, D. J., J. B. Cucchiaro, and S. M. Sherman (1987) Innervation of the cat's lateral geniculate nucleus by individual cells of the perigeniculate nucleus. Soc. Neurosci. Abstr. 13: 1435.

Updyke, B. V. (1975) Patterns of projections of cortical areas 17, 18 and 19 onto the dorsal lateral geniculate nucleus in the cat. J. Comp. Neurol. 163: 377-396. 